\title{
The Symmetry of the Flow of Newtonian and Non-Newtonian Fluid in a Plane Diffuser and Confusor
}

\author{
A.I. Fedyushkin, E.V. Volkov \\ Ishlinsky Institute for Problems in Mechanics of Russian Academy of Sciences, \\ Moscow, 119526, Russia \\ evvolkov94@mail.ru, fai@ipmnet.ru
}

\begin{abstract}
In the paper the results of studying the various laminar flow regimes in a plane diffuser with a small opening angle obtained for a viscous incompressible fluid by numerical solving the $\mathrm{Na}-$ vier-Stokes equations are presented. A transition of the flow regimes in a diffuser from a symmetric stationary regime to asymmetric stationary one and next to asymmetric non-stationary regime in their dependence on the Reynolds number is demonstrated. The values of Reynolds number that define the ranges of existence of a given regime are pointed out.

The paper presents the results on the change in the nature of flows from stationary - symmetric to stationary - asymmetric and to non-stationary in the diffuser and confusor depending on the Reynolds number. The ranges of existence of these flow regimes in plane diffusers and confusers depending on the Reynolds number (flow rate) for Newtonian, pseudo plastic and dilatants fluids with the Ostwald-de Waale power law for viscosity are numerically found.

The results of comparison of numerical simulation of laminar viscous fluid flows in a plane diffuser and confusor for symmetric and asymmetric boundary conditions at the inlet are presented. The analysis of the obtained results allows us to conclude that there is a significant difference between the modes of symmetric fluid flows in the diffuser and the confusor.
\end{abstract}

Keywords: numerical simulation, diffuser, confusor, asymmetric flow. 
УДК 532.517 .3

\title{
Симметрия течения ньютоновской и неньютоновской жидкости в плоском диффузоре и конфузоре
}

\author{
А.И. Федюшкин, Е. В. Волков \\ Институт проблем механики им. А.Ю. Иилинского РАН, Россия, Москва, 119526, \\ проспект Вернадского, д. 101, корп. 1 \\ fai@ipmnet.ru, evvolkov94@mail.ru
}

\begin{abstract}
Аннотация
Представлены результаты исследования различных режимов ламинарного течения в плоском диффузоре/конфузоре с малым углом раствора. Результаты получены для вязкой несжимаемой жидкости путем численного моделирования на основе решения уравнений Навье-Стокса. Найдены области существования и переходы режимов течения от стационарно-симметричных к стационарно-асимметричным и к нестационарным в диффузоре и конфузоре в зависимости от числа Рейнольдса. Приведены значения числа Рейнольдса, определяющие диапазоны существования этих режимов течения жидкости для ньютоновских и неньютоновских жидкостей.
\end{abstract}

Ключевые слова: симметрия течения, диффузор, конфузор, моделирование.

\section{1. Введение}

В работе рассматриваются задачи ламинарного течения ньютоновских и неньютоновских вязких несжимаемых жидкостей в плоском диффузоре и конфузоре с малым углом раствора. Данная задача имеет, как фундаментальное научное значение для гидромеханики, так и прикладные приложения во многих процессах в разных отраслях промышленности и сфеpax деятельности человека, например, таких, как авиация, космонавтика, машиностроение, нефтегазодобыча, медицина и фармакология, гидрология рек и океана, теплоэнергетика и др. Рассматриваемая задача аналогична классической задаче Джеффри-Гамеля (ДГ) о течении вязкой несжимаемой жидкости в диффузоре/конфузоре при различных числах Рейнольдса [1, 2]. Наша постановка отличается от задачи ДГ тем, что, во-первых, мы рассматриваем конечную геометрию плоского диффузора/конфузора и решение ищется численно на основе решения полных уравнений Навье-Стокса для несжимаемой вязкой жидкости, во-вторых, наша постановка не накладывает ограничений на существование тангенсальных скоростей и асимметрии течения. Течение несжимаемой жидкости в диффузоре и конфузоре отличается от течения в канале с параллельными стенками, в связи с расширением/сужением области течения, где кинетическая энергия потока преобразуется в статическое давление. В диффузоре течение жидкости осуществляется навстречу градиенту давления, а в конфузоре - поток направлен в одну сторону с градиентом давления. Это две разные ситуации, что принципиально влияет на возникновение бифуркаций, неустойчивость пограничного слоя и нарушение симметрии течения [3-5]. Несмотря на то, что задача ДГ проста в постановке, определяется двумя параметрами (углом раствора и числом Рейнольдса) [1, 2], она насыщена гидродинамическими явлениями и особенностями, и поскольку она имеется множество приложений, то изучение решений задачи ДГ актуально по сей день. Существует много работ по исследованию течений несжимаемой жидкости в диффузорах и конфузорах. Следует отметить, что большая часть этих работ относится к изучению нестационарных и турбулентных режимов 
течения, а также к задачам с дополнительными геометрическими и физическими особенностями в постановке, отличающимися от классической задачи ДГ. Среди работ о течениях в плоских диффузорах и конфузорах можно выделить работы [1-22]. Детали и особенности задачи ДГ описаны и обсуждены в книгах [3, 4]. В статье [5] представлен обзор более 150 работ по изучению решений задачи ДГ, изучается асимптотический характер решений и дано обобщение решений ДГ на основе группового анализа дифференциальных уравнений. Ранние исследования течения ДГ были проведены в работе [6], где было получено решение стационарной двумерной задачи ДГ с использованием эллиптической функции Якоби. В работах $[7,8]$ найдены обобщения решения задачи ДГ, указано на наличие несимметричных стационарных течений и приведены одно -, двух- и трехмодовые бифуркационные решения. Эти исследования свидетельствуют о наличии стационарных асимметричных многомодовых решений для определенных диапазонов чисел Рейнольдса и углов раствора диффузора.

Течения струй в открытое пространство и течения во внезапно расширяющиеся каналы можно рассматривать как предельный случай течения в диффузорах с углами раствора 360 или 180 градусов соответственно. Исследование нарушения симметрии течения и бифуркаций в задаче ДГ, включающей течение в канале и предельный случай диффузора с углом раскрытия 180 градусов, проводилось в работе [9]. Результаты экспериментальных и теоретических исследований течения в симметричном внезапно расширяющемся канале представлены в работах [10-14]. В работе [10] представлены картины течения и профили скоростей в канале с мгновенным симметричным расширением канала, измеренные лазерно-доплеровским методом. В работе [10] экспериментально показано, что при малых числах Рейнольдса $(\mathrm{Re}=114$ и $\mathrm{Re}=256)$ в симметричном канале со ступенчатым расширением, течение может иметь стационарный и асимметричный характер. Кроме этого в [10] указывается на слабое проявление в скорости течения трехмерности при малых числах Рейнольдса. В [11] показано, что при малых числах Рейнольдса флуктуации энергии в канале могут превышать флуктуации энергии, вызванные турбулентностью. В работах [11-17] представлены исследования возникновения бифуркаций в ДГ течениях жидкости, приведены критические значения чисел Рейнольдса возникновения различных типов бифуркаций и нейтральные кривые (число Рейнольдса / отношение сторон плеча расширения) для стационарных, периодических, симметричных и несимметричных течений в каналах с внезапным расширением. Появление стационарных и нестационарных волн при течении жидкости в диффузоре в диапазоне чисел Рейнольдса от 5 до 5000 при различных углах раствора диффузора было изучено в работе [15]. Приведены зависимости и асимптотика существования волн в зависимости от угла раствора диффузора и числа Рейнольдса. В работе [17] результаты 2D и 3D (с условием симметрии) численного моделирования течения вязкой несжимаемой жидкости для чисел Рейнольдса в диапазоне от 60 до 360 и угла раствора от $10^{\circ}$ до $180^{\circ}$ в плоском диффузоре при наличии входного участка. Авторы работы [17] показали влияние трехмерности и колебаний скорости на входе диффузора на наличие нестационарных несимметричных режимов течения. Результаты расчетов авторов работы [18] показали, что в областях конечных размеров также могут существовать устойчивые во времени стационарные решения в виде аналогов стоячих волн, существование которых было показано ранее в работе [15] для бесконечных областей. В работе [18] также показано, что в некоторых случаях существует неединственность устойчивых решений.

Распространение традиционной задачи ДГ на течения в плоских каналах с растягивающимися сходящимися / расходящимися стенками было сделано в работах $[19,20]$. В этих работах показано влияние угла раскрытия и скорости растяжения стенок на характеристики течения жидкости. В работе [21] рассмотрена задача численного моделирования нестационарных дозвуковых течений вязкого газа в канале с внезапным симметричным расширением поперечного сечения (диффузора). В широком диапазоне характеристических параметров численно исследованы нелинейные процессы развития неустойчивости рассматриваемого 
течения с учетом акустико-вихревых взаимодействий. Обнаружены эффекты звукового самовозбуждения струи, текущей в широкую часть канала. Оценено влияние входных профилей средней скорости на эволюцию течения газа.

Известно, что характер течения вязкой жидкости в плоском двумерном диффузоре / конфузоре определяется геометрией (углом расширения/сужения каналов $\beta$ ) и числом Рейнольдса. Угол раствора в диффузоре начинает оказывать существенное влияние на характеристики потока при превышении десяти градусов $[7,15,18-20]$, поэтому в данной работе моделирование течений жидкости в диффузоре/конфузоре проводилось для геометрии с малым углом раствора, равным четырем градусам. В работе [22] представлены результаты, основанные на численном решении задачи ДГ для диффузора с малым углом раскрытия и показано изменение режимов течения в диффузоре в зависимости от числа Рейнольдса от симметричного стационарного к несимметричному стационарному и к нестационарному несимметричному. В работе [22] указаны значения диапазонов чисел Рейнольдса существования этих режимов течения, что согласуется с наличием различных мод в решениях задачи ДГ, показанных в работах $[7,8]$.

Данная работа является продолжением работы [22] и представляет результаты по изучению изменения характера течений (от стационарно-симметричных к стационарно-асимметричным и к нестационарным) в диффузоре и конфузоре в зависимости от числа Рейнольдса. Численно определены диапазоны существования этих режимов течения в плоских диффузорах и конфузорах в зависимости от числа Рейнольдса (расхода) для ньютоновской, псевдопластичной и дилатантной жидкостей со степенным законом Оствальда-де-Ваале для вязкости $[24,25]$. В настоящей работе представлены результаты сравнения численного моделирования ламинарных течений вязкой жидкости в плоском диффузоре и конфузоре для симметричных и несимметричных граничных условий на входе. Анализ полученных результатов позволяет сделать вывод о принципиальном различии режимов симметричных течений жидкости в диффузоре и конфузоре.

\section{2. Постановка задачи}

Рассматривается ламинарное течение вязкой несжимаемой жидкости между двумя плоскими стенками, наклонёнными друг к другу под небольшим углом, то есть течение Джеффри - Гамеля в плоском диффузоре (рис. 1) и конфузоре (рис. 2). Целью численного моделирования является определение диапазонов существования симметричных и несимметричных стационарных течений и переход к нестационарному режиму для псевдопластичной, ньютоновской и дилатантной жидкостей в диапазоне чисел Рейнольдса $\operatorname{Re}<500$, и провести сравнение полученных результатов моделирования.

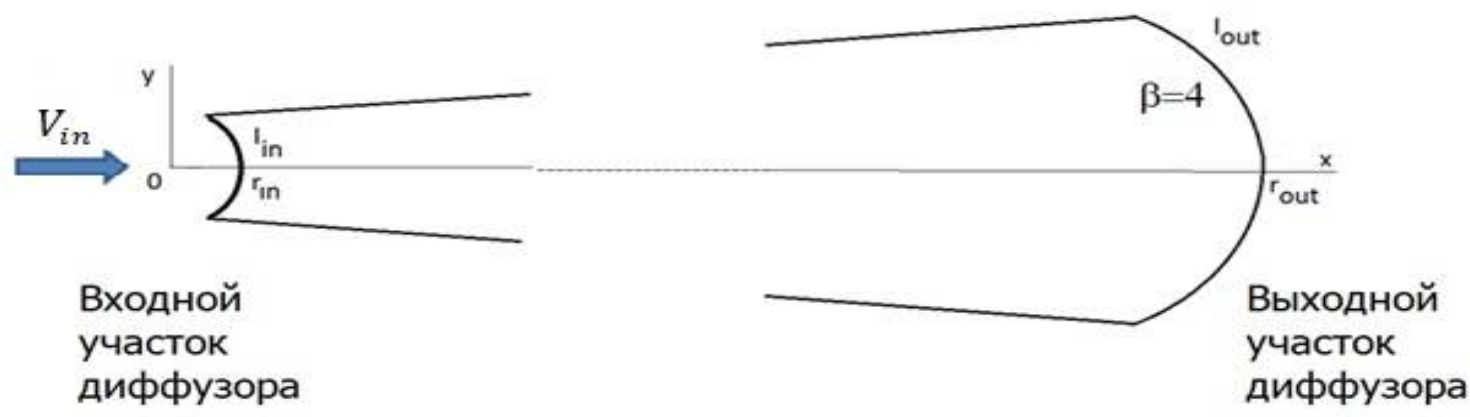

Рис. 1. Схема плоского диффузора расчетной области с углом раствора $\beta=4^{\circ}$ и длиной $L=0.495$ метра 
Геометрическая модель диффузора: угол раствора диффузора $\beta=4^{\circ}$, входная граница имеет форму дуги $l_{\text {in }}\left(r_{\text {in }}=0.005\right.$ м, где $\left.r^{2}=x^{2}+y^{2}\right)$, выходная граница имеет форму дуги $l_{\text {out }}\left(r_{\text {out }}=0.5 \mathrm{M}\right.$, где $\left.r^{2}=x^{2}+y^{2}\right)$. Длина диффузора равна $L=\left|r_{\text {out }}-r_{\text {in }}\right|=0.495$ метра.

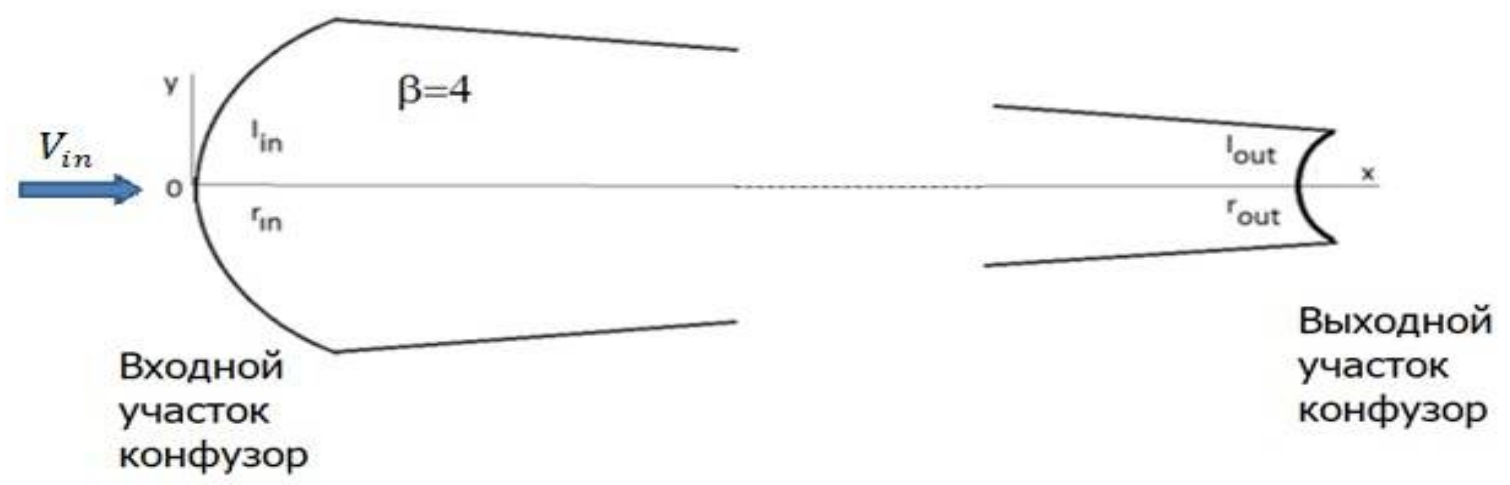

Рис. 2. Схема плоского конфузора расчетной области с углом раствора $\beta=4^{\circ}$ и длинной $L=0.495$ метра

Геометрическая модель конфузора следующая: угол раствора конфузора $\beta=4^{\circ}$, входная граница имеет форму дуги $l_{\text {in }}\left(r_{\text {in }}=-0.5 \mathrm{M}\right.$, где $\left.r^{2}=x^{2}+y^{2}\right)$, выходная граница имеет форму дуги $l_{\text {out }}\left(r_{\text {out }}=-0.005 \mathrm{M}\right.$, где $\left.r^{2}=x^{2}+y^{2}\right)$. Длина конфузора равна $L=\left|r_{\text {out }}-r_{\text {in }}\right|=$ $=0.495$ м. Входные и выходные границы не были плоскими, что показано на рис. 1 и рис. 2 , где изображения кривизны этих границ намеренно искажены в большую сторону.

Геометрические модели диффузора и конфузора были намеренно выбраны с большим отношением сторон (приблизительно 50), чтобы проанализировать течение в диффузоре (конфузоре) вдали от входных и выходных участков. Угол раствора был выбран менее десяти градусов, чтобы исключить его влияние на характер течения.

Моделирование задачи осуществляется на основе численного решения системы уравнений Навье - Стокса для несжимаемой вязкой жидкости

$$
\begin{gathered}
\frac{\partial V}{\partial t}+(V \nabla) V=-\frac{\nabla P}{\rho}+\frac{1}{\rho} \nabla \sigma(V), \\
\operatorname{div}(\mathbf{V})=0
\end{gathered}
$$

где $V=V\left(v_{x}, v_{y}\right)$ - вектор скорости; $P$ - давление; $\rho$ - плотность; $\sigma(V)$ - тензор вязких напряжений.

В качестве граничных условий, на входе в диффузор/конфузор, задан постоянный положительный расход $Q$ (число Рейнольдса $\operatorname{Re}$ ), на выходе задано давление $P=0$. На верхней и нижней границе для скорости ставится условие прилипания: $V=0$.

Число Рейнольдса на входе в диффузор определено, как

$$
\operatorname{Re}=\frac{V_{i n} l_{\text {in }}}{v},
$$

где $l_{\text {in }}$ - длина дуги на входе в диффузор (конфузор); $V_{\text {in }}$ - скорость на входе в диффузор (конфузор), направленная по нормали к границе, соответствующая расходу $Q$.

Моделирование проводилось для ньютоновской, псевдопластичной и дилатантной жидкостей со степенным законом Оствальда-де Ваале для вязкости [24]. В одномерном случае вязкое напряжение для этого закона можно записать в виде

$$
\tau=k\left(\frac{\partial u}{\partial y}\right)^{n},
$$


где $k>0$ - коэффициент густоты потока (в СИ единицей измерения служит Па $c^{n}$ ); $u-$ скорость течения; $y$ - координата перпендикулярная направлению течения. Величину, как функцию градиента скорости, определенную по формуле

$$
\mu_{\text {eff }}=k\left(\frac{\partial u}{\partial y}\right)^{n-1},
$$

в литературе часто называют эффективной вязкостью (в СИ измеряется в Па $с$ ). Для двумерного случая компоненты тензора вязкого напряжения для степенного закона Оствальдаде Вааля можно найти, например, в [24, 25]. Типы жидкостей с вязкостями, соответствующими степенному закону вязкости Оствальда-де Ваале [25], приведены в табл. 1.

\section{Таблица 1}

\section{Тип жидкости в зависимости от показателя степени $n$}

\begin{tabular}{c|c}
\hline$n$ & Тип жидкости \\
\hline$n<1$ & Псевдопластичная \\
$n=1$ & Ньютоновская \\
$n>1$ & Дилатантная \\
\hline
\end{tabular}

Численное решение двумерных уравнений Навье-Стокса осуществлялась методом контрольных объёмов [23], использовались схемы второго и третьего порядка точности по пространству и первого порядка по времени. Тестовые расчеты проводились на последовательности сеток с уменьшающимся шагом сетки. Из данных тестов были выбраны расчетные сетки с таким количеством узлов сетки, при увеличении которых (при измельчении сетки) результаты практически не отличались. Численные вычисления получены на сетках с запасом точности для предотвращения появления численной неустойчивости (величины сеточных чисел Рейнольдса и значения чисел Куранта были не более единицы). При моделировании использовались подробные неравномерные сетки, и проводился контроль точности на каждом шаге по времени. Ячейки сетки вблизи твердых продольных стенок были прямоугольными и ортогональными. Использовались неравномерные сетки с уменьшающимся шагом сетки около входа и выхода в диффузор/конфузор, а также вблизи твердых продольных границ (в пограничных слоях было не менее десяти узлов сетки). Около твердых стенок (в пограничных слоях) сетка была ортогональна на расстоянии нескольких десятков шагов сетки. Кроме того, для различных сеток, для определения достаточности размера сетки проводилось сравнение профилей скоростей вдоль линий, параллельным стенкам на небольшом расстоянии от твердых стенок $(0,1$ размера входной части диффузора и параллельно твердым стенкам). Для заданной скорости на входе в диффузор/конфузор расчеты выполнялись от нулевых начальных скоростей в расчетной области до установления стационарного (или квазистационарного) режима течения. Анализ численных решений и характера течения проводился на установившемся стационарном или квазистационарном режиме.

\section{3. Результаты численного моделирования}

В предыдущих работах [22] были получены следующие диапазоны чисел Рейнольдса для режимов течения несжимаемой жидкости (ньютоновской) в плоском диффузоре с углом раствора $\beta=4^{\circ}$, полученные из результатов численного моделирования (табл. 2).

Результаты о характере течения ньютоновской и неньютоновской жидкостей в диффузоре и конфузоре с углом раствора $\beta=4^{\circ}$, полученные на основе численного моделирования приведены в табл. 3 и 4. 
Таблий 2

Тип течения в плоском диффузоре в зависимости от числа Рейнольдса

\begin{tabular}{c|c|c}
\hline $\begin{array}{c}\text { ССТ - стационарное } \\
\text { симметричное течение }\end{array}$ & $\begin{array}{c}\text { СНТ - стационарное } \\
\text { несимметричное течение }\end{array}$ & $\begin{array}{c}\text { ННТ - нестационарное } \\
\text { несимметричное течение }\end{array}$ \\
\hline $\operatorname{Re}<270$ & $270<\operatorname{Re}<350$ & $\operatorname{Re}>400$ \\
\hline
\end{tabular}

Таблица 3

Результаты численного моделирования для диффузора

\begin{tabular}{|c|c|c|c|c|}
\hline \multicolumn{5}{|c|}{ Псевдопластичная $(n=0.5)$} \\
\hline$Q_{\text {input }}[\kappa \Gamma / \mathrm{c}] \quad\left(\mathrm{Re}_{\text {in }}\right)$ & $0.05(50)$ & $0.1(100)$ & $0.3(300)$ & $0.5(500)$ \\
\hline Тип течения & сст & сст & снт & ннт \\
\hline \multicolumn{5}{|c|}{ Ньютоновская $(n=1)$} \\
\hline$Q_{\text {input }}[\mathrm{\kappa} \Gamma / \mathrm{c}] \quad\left(\mathrm{Re}_{\text {in }}\right)$ & $0.05(50)$ & $0.1(100)$ & $0.3(300)$ & $0.5(500)$ \\
\hline Тип течения & сст & $\mathrm{ccT}$ & снт & ннт \\
\hline \multicolumn{5}{|c|}{ Дилатантная ( $n=2)$} \\
\hline$Q_{\text {input }}[\mathrm{\kappa} \Gamma / \mathrm{c}] \quad\left(\mathrm{Re}_{\text {in }}\right)$ & $0.05(50)$ & $0.1(100)$ & $0.3(300)$ & $0.5(500)$ \\
\hline Тип течения & $\mathrm{ccT}$ & сст & $\mathrm{cct}$ & сст \\
\hline
\end{tabular}

Таблица 4

Результаты численного моделирования для конфузора

\begin{tabular}{|c|c|c|c|}
\hline \multicolumn{4}{|c|}{ Псевдопластичная $(n=0.5)$} \\
\hline$Q_{\text {input }}[\mathrm{\kappa} \Gamma / \mathrm{c}] \quad\left(\mathrm{Re}_{\text {in }}\right)$ & $0.05(50)$ & $0.1(100)$ & $0.5(500)$ \\
\hline Тип течения & $\mathrm{ccT}$ & $\mathrm{cct}$ & $\mathrm{ceT}$ \\
\hline \multicolumn{4}{|c|}{ Ньютоновская $(n=1)$} \\
\hline$Q_{\text {input }}[\mathrm{\kappa} / \mathrm{c}] \quad\left(\mathrm{Re}_{\text {in }}\right)$ & $0.05(50)$ & $0.1(100)$ & $0.5(500)$ \\
\hline Тип течения & $\mathrm{ccT}$ & сст & сcт \\
\hline \multicolumn{4}{|c|}{ Дилатантная $(n=2)$} \\
\hline$Q_{\text {input }}[\mathrm{\kappa} / \mathrm{c}] \quad\left(\mathrm{Re}_{\text {in }}\right)$ & $0.05(50)$ & $0.1(100)$ & $0.5(500)$ \\
\hline Тип течения & $\mathrm{ceT}$ & $\mathrm{cct}$ & $\mathrm{ceT}$ \\
\hline
\end{tabular}

\section{1. Стационарные симметричные течения}

Численные результаты показали, что для рассмотренных вариантов расчетов, указанных в табл. 3, для псевдопластичной, ньютоновской и дилатантной жидкостей в плоском диффузоре численно показан диапазон существования стационарных симметричных течений. Но также видно, что для разных типов жидкости режим держится до разных чисел Рейнольдса. Из сравнения результатов, представленных в табл. 4, можно сделать вывод, что в рассмотренном диапазоне чисел Рейнольдса $(\operatorname{Re}<500)$ в плоском конфузоре, течения являются стационарными и симметричными, стационарного режима с несимметричной структурой течений не наблюдается. 
Результаты численного моделирования течения в диффузоре представлены на рис. 3 и рис. 4. На рис. $3, a, \sigma, 6$ представлены изолинии горизонтальной компоненты скорости, а также в увеличенном масштабе для входного участка диффузора, отмеченного овалом. На этих же рисунках показаны изолинии угла вектора скорости:

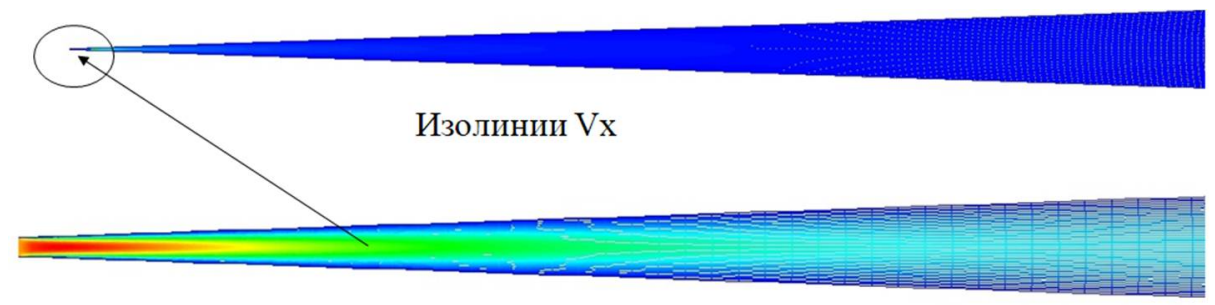

Изолинии Vx (входной участок диффузора)

Изолинии угла вектора скорости $\operatorname{arctg}(\mathrm{Vx} / \mathrm{Vy})$

Рис. 3, $a$. Изолинии горизонтальной компоненты вектора скорости и угла вектора скорости течения псевдопластичной жидкости в диффузоре $(Q=0.1[\kappa г / \mathrm{c}], \operatorname{Re}=100, n=0.5)$

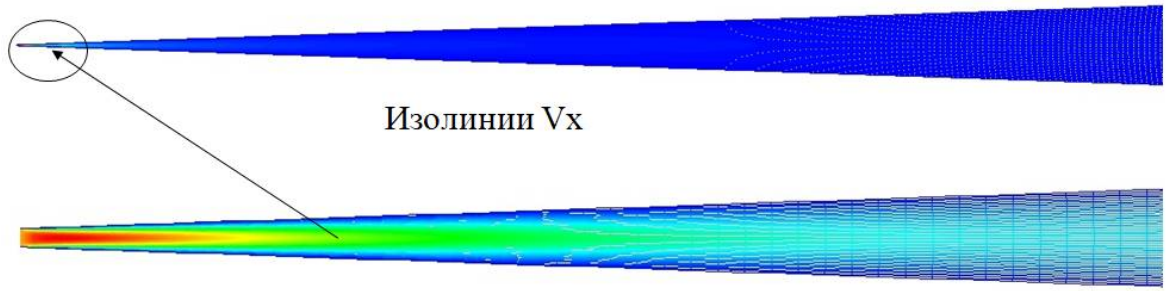

Изолинии Vx (входной участок диффузора)

Изолинии угла вектора скорости $\operatorname{arctg}(\mathrm{Vx} / \mathrm{Vy})$

Рис. 3, б. Изолинии горизонтальной компоненты вектора скорости и угла вектора скорости течения ньютоновской жидкости в диффузоре ( $Q=0.1[\kappa г / \mathrm{c}], \operatorname{Re}=100, n=1)$

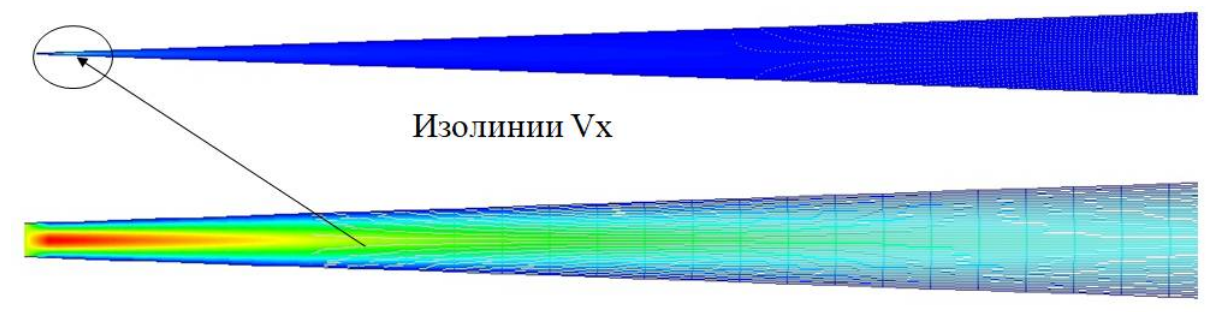

Изолинии Vx (входной участок диффузора)

Изолинии угла вектора скорости $\operatorname{arctg}(\mathrm{Vx} / \mathrm{Vy})$

Рис. 3, в. Изолинии горизонтальной компоненты вектора скорости и угла вектора скорости течения дилатантной жидкости в диффузоре $(Q=0.5[$ [кг/с], $\operatorname{Re}=500, n=2)$

В данной работе приняты следующие обозначения безразмерных величин:

$$
V_{\text {mag_dimless }}=\frac{|V|}{V_{\max }}, \quad y_{\text {dimless }}=\frac{y}{(0.005-x)^{*} 0.035}
$$


На рис. 4, $a, 6,6$ показаны безразмерные профили скорости в четырех вертикальных сечениях для диффузора $x=0.1,0.2,0.3,0.4 \mathrm{M}$.

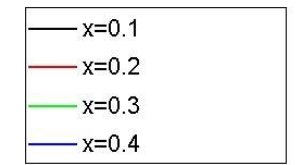

vmag_dimless

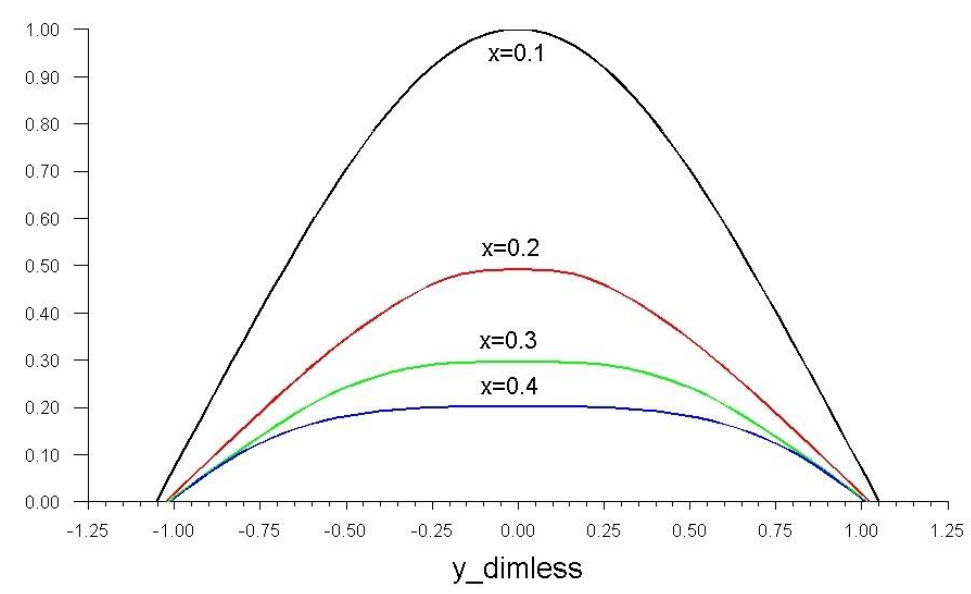

Рис. 4, $a$. Профили скорости течения псевдопластичной жидкости в диффузоре $(Q=0.1$ $[\kappa г / \mathrm{c}], \operatorname{Re}=100, n=0.5$, в сечениях $x=0.1,0.2,0.3,0.4 \mathrm{M})$

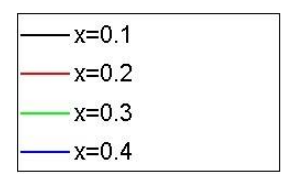

vmag_dimless

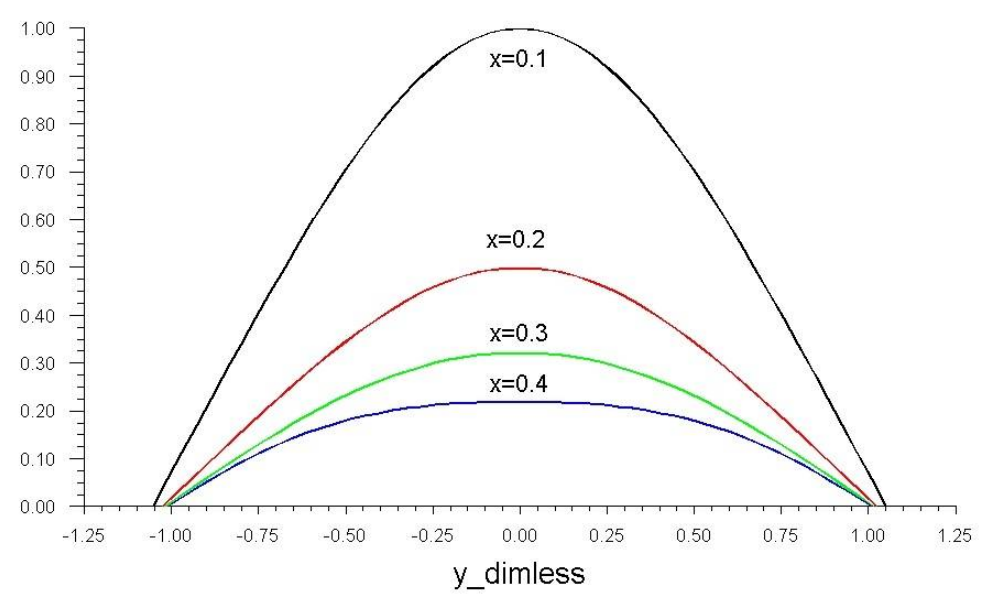

Рис. 4, б. Профили скорости течения ньютоновской жидкости в диффузоре ( $Q=0.1[\kappa \Gamma / \mathrm{c}]$, $\mathrm{Re}=100, n=1$, в сечениях $x=0.1,0.2,0.3,0.4 \mathrm{~m}$ )

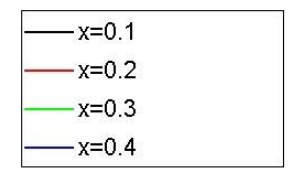

vmag_dimless

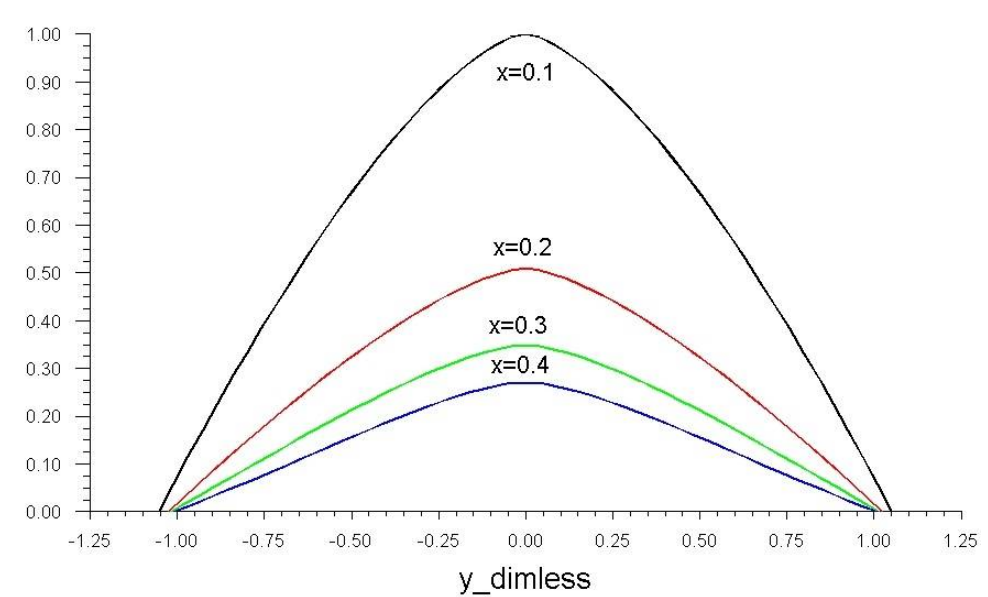

Рис. 4 , в. Профили скорости течения дилатантной жидкости в диффузоре ( $Q=0.5$ [кг/с], $\operatorname{Re}=500, n=2$, в сечениях $x=0.1,0.2,0.3,0.4 \mathrm{M})$ 
Результаты моделирования течения псевдопластичной $(n=0.5)$ и дилатантной жидкости в конфузоре $(n=2)$ жидкостей в конфузоре для $\mathrm{Re}=500$ представлены на рис. 5 и рис. 6. На рис. 5, $а$ и рис. 5, б представлены изолинии горизонтальной компоненты скорости во всей расчетной области, а также в увеличенном масштабе около входного участка конфузора, отмеченного овалом. На этих же рисунках внизу показаны изолинии угла вектора скорости $\operatorname{arctg}\left(V_{x} / V_{y}\right)$.

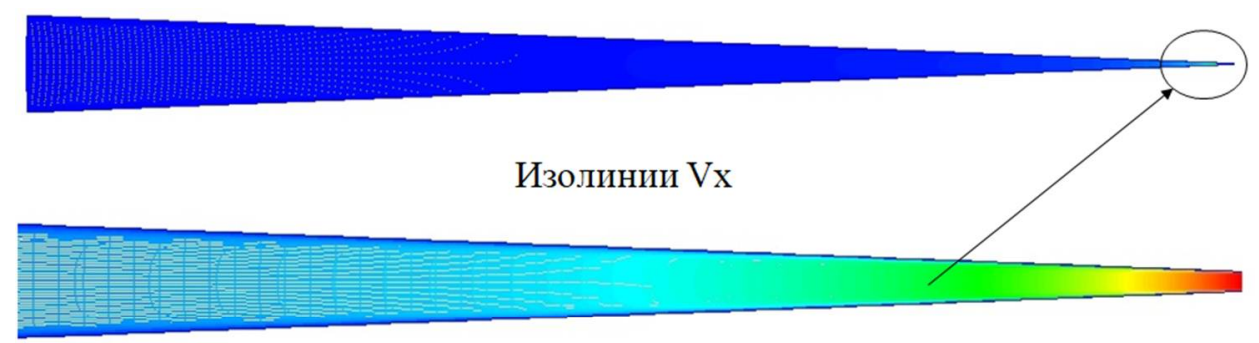

Изолинии Vx (входной участок конфузора)

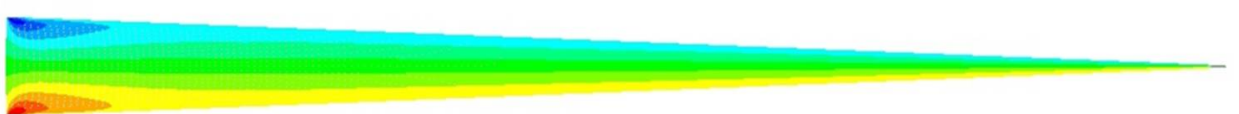

Изолинии угла вектора скорости $\operatorname{arctg}(\mathrm{Vx} / \mathrm{Vy})$

Рис. 5, $a$. Изолинии горизонтальной компоненты вектора скорости и угла вектора скорости течения псевдопластичной жидкости в конфузоре $(Q=0.5[\kappa г / \mathrm{c}], \operatorname{Re}=500, n=0.5)$

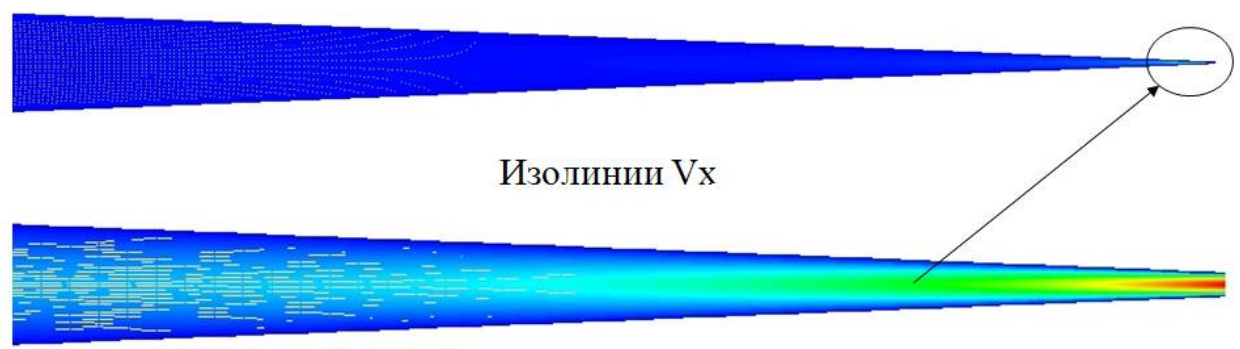

Изолинии Vx (входной участок конфузора)

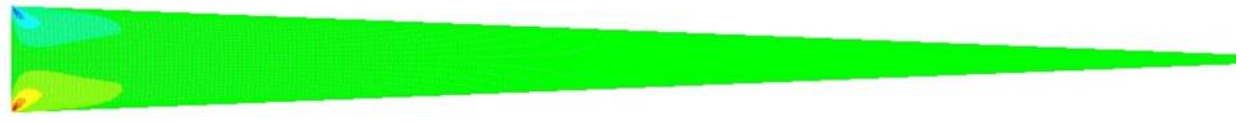

Изолинии угла вектора скорости $\operatorname{arctg}(\mathrm{Vx} / \mathrm{Vy})$

Рис. 5, б. Изолинии горизонтальной компоненты вектора скорости и угла вектора скорости течения дилатантной жидкости в конфузоре $(Q=0.5[\kappa г / \mathrm{c}], \operatorname{Re}=500, n=2)$

На рис. $6, a, \sigma$ для течения $(Q=0.5[$ [кг/с], $\mathrm{Re}=500)$ псевдопластичной $(n=0.5)$ и дилатантной $(n=2)$ жидкостей в конфузоре представлены безразмерные профили модуля скорости в четырех вертикальных сечениях $x=0.1,0.2,0.3,0.4$ м. Сравнение изолиний, изображенных на рис. 5, $a$ и рис. 5, 6 , а также профилей скорости для псевдопластичной (рис. $6, a)$ и для дилатантной жидкости (рис.6,б) показывает существенное различие в характере течения и влияние на него вязкости. Течение псевдопластичной $(n=0.5)$ жидкости характеризуется тонкими пограничными слоями и плоским профилем скорости, а течение дилатантной жидкости вдали от входа $(x>0.1)$ имеет увеличенные пограничные слои с чётно степенным характером профиля скорости. Следует отметить, что в обоих случаях течения обладают радиальной симметрией. 


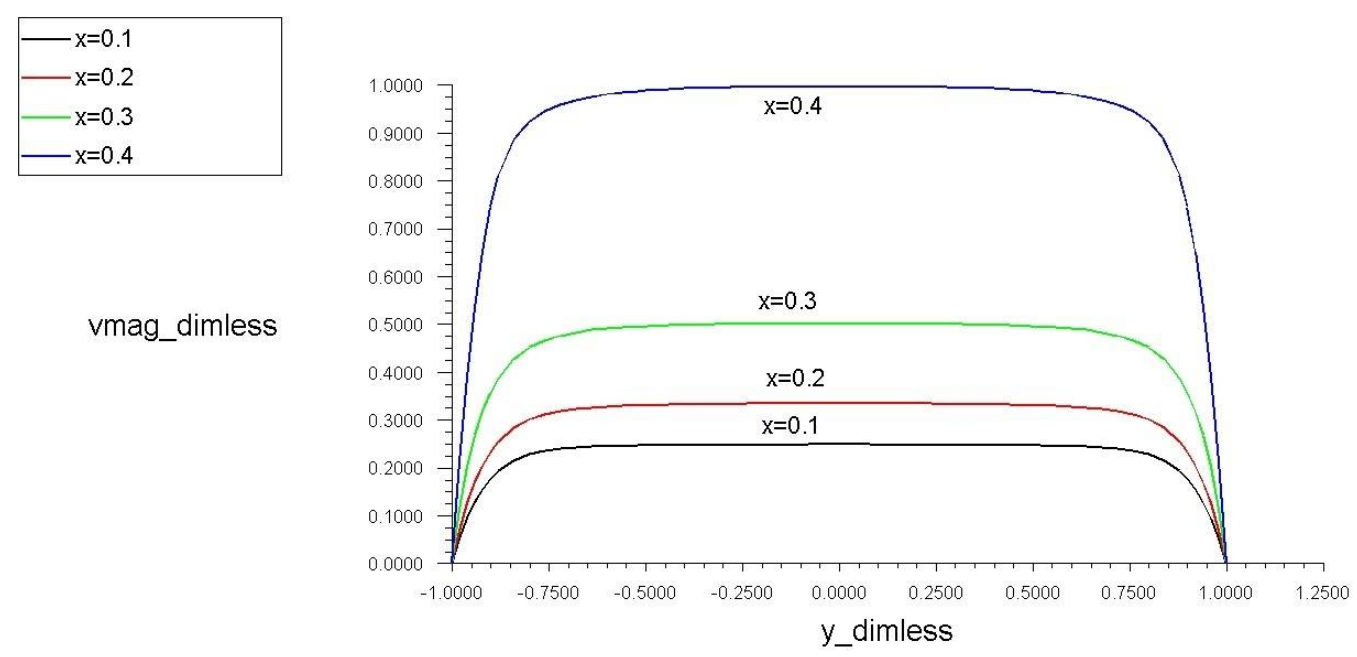

Рис. 6, $a$. Профили скорости течения псевдопластичной жидкости в конфузоре ( $Q=0.5$ $[\kappa г / \mathrm{c}], \operatorname{Re}=500, n=0.5$, в сечениях $x=0.1,0.2,0.3,0.4 \mathrm{M}$ )

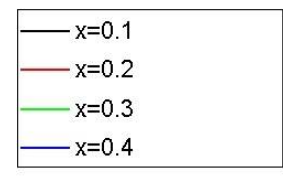

vmag_dimless

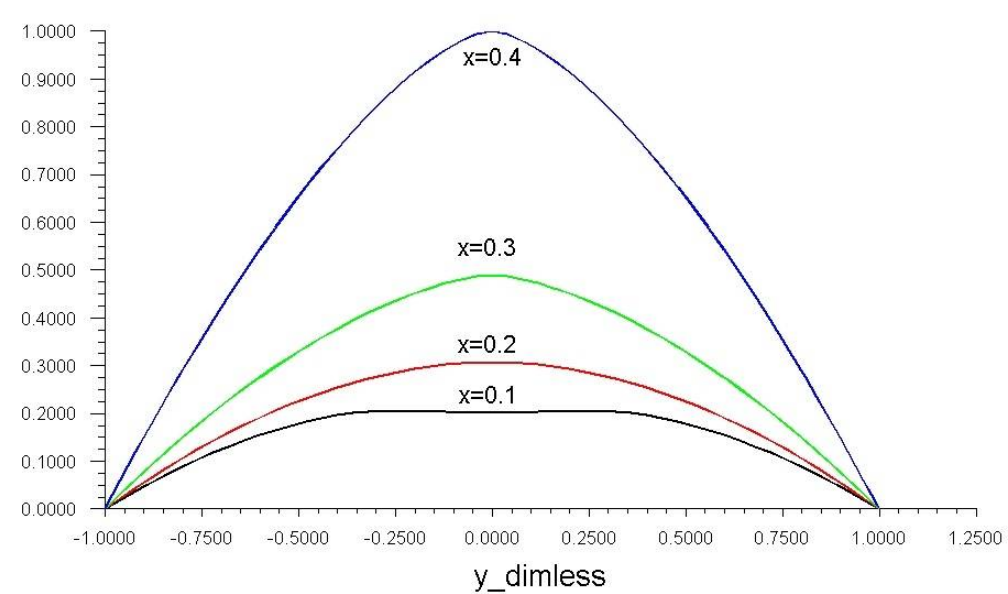

Рис. 6, б. Профили скорости течения дилатантной жидкости в конфузоре ( $Q=0.5$ [кг/с], $\operatorname{Re}=500, n=2$, в сечениях $x=0.1,0.2,0.3,0.4 \mathrm{M})$

Результаты моделирования течения в диффузоре и конфузоре, показали, что при одинаковых условиях течение в конфузоре более устойчивое, чем в диффузоре и остается стационарным симметричным в более широких диапазонах числа Рейнольдса.

\section{2. Стационарные несимметричные течения}

Результаты расчетов стационарных несимметричных течений, полученные для диффузора, подтверждают тот факт, что при числе Рейнольдса Re превосходящем некоторое критическое значении Re* происходит нарушение симметрии в структуре течения, с образованием вихревых структур с возвратными течениями около твердых стенок диффузора, это известный факт из многочисленной литературы, например, [3, 4]. Для течения несжимаемой жидкости диффузоре в работе [7] было найдено критическое число равное $\mathrm{Re}^{*}=269$, наличие которого затем численно было подтверждено в работе [22]. Численные расчеты данной работы показали, что, при увеличении значения числа Рейнольдса выше критического происходит срыв пограничного слоя и на определенном расстоянии от входа в диффузор, вблизи продольных стенок диффузора формируются слабые возвратные течения, как для псевдопластичной $(n=0.5)$, так и для ньютоновской $(n=1)$ жидкости. Неустойчивость с образованием вторичных вихрей на разных стенах диффузора может начинаться на разном расстоянии от входа в диффузор, что приводит к несимметрии течения. В зонах отрыва пограничного слоя 
вектор скорости меняет направление. Численные расчеты показали, что течения для псевдопластичной $(n=0.5)$ и для ньютоновской $(n=1)$ жидкости в диффузоре перестают быть симметричным, оставаясь стационарным в узком диапазоне чисел Рейнольдса $300>\operatorname{Re}>270$.

На рис. 7 представлены изолинии горизонтальной компоненты скорости для всей расчетной области, а также в увеличенном масштабе около входного участка диффузора, отмеченного овалом. На этих же рисунках показаны изолинии угла вектора скорости.

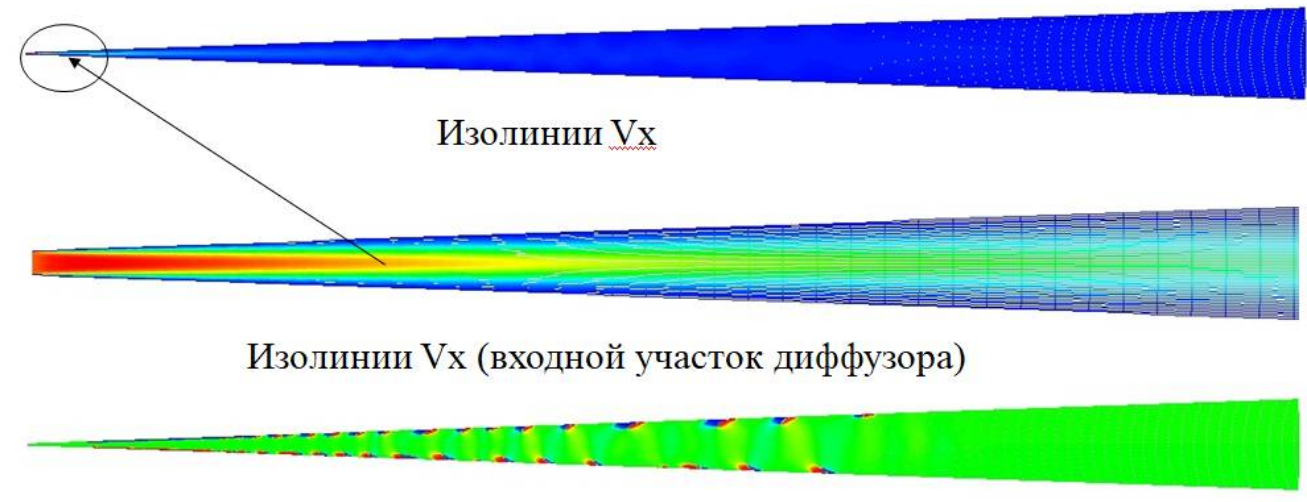

Изолинии угла вектора скорости $\operatorname{arctg}(\mathrm{Vx} / \mathrm{Vy})$

Рис. 7. Изолинии горизонтальной компоненты вектора скорости и угла вектора скорости течения псевдопластичной жидкости в диффузоре ( $Q=0.3[$ [кг $/ \mathrm{c}], \operatorname{Re}=300, n=0.5)$

На рис. 8 показаны безразмерные профили скорости в четырех вертикальных сечениях для диффузора $x=0.1,0.2,0.3,0.4 \mathrm{м}$.
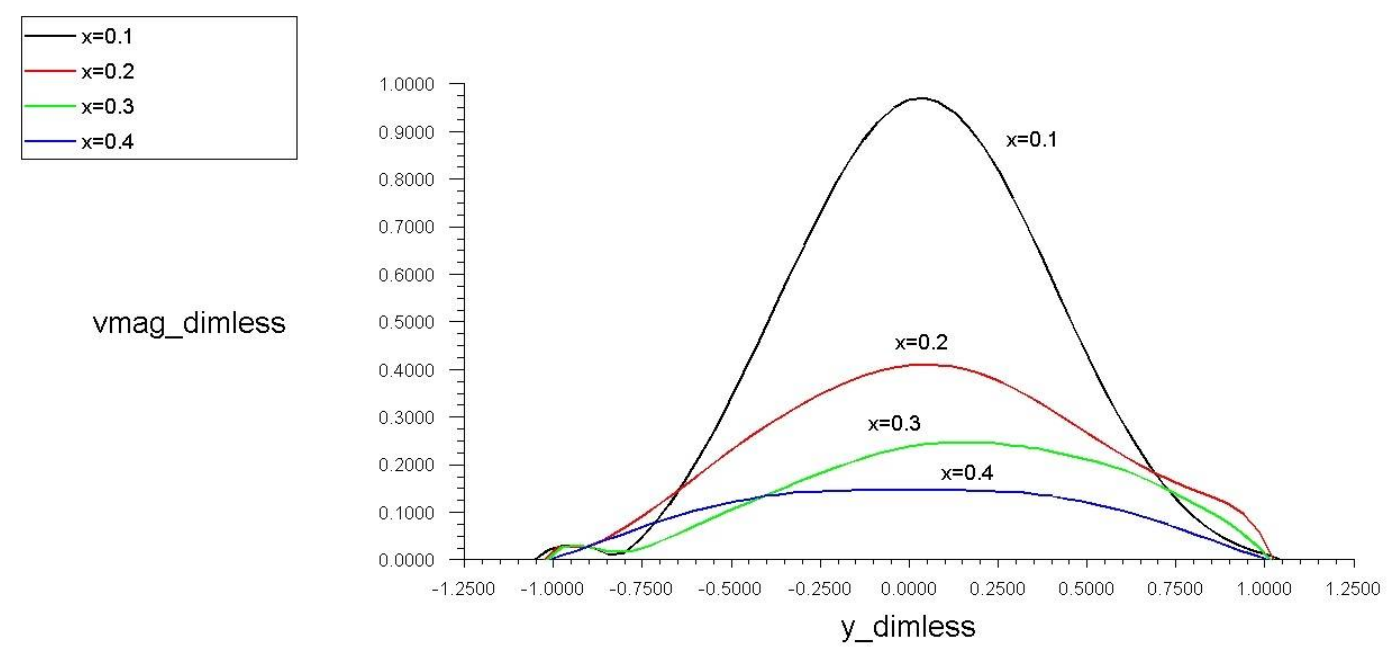

Рис. 8. Профили скорости течения псевдопластичной жидкости в диффузоре ( $Q=0.3$ $[\kappa \Gamma / \mathrm{c}], \operatorname{Re}=300, n=0.5$, в сечениях $x=0.1,0.2,0.3,0.4 \mathrm{M}$ )

\section{3. Нестационарные несимметричные течения}

С увеличением числа Рейнольдса интенсивность вихревых вторичных пристеночных течений возрастает, картина их расположения в продольном направлении образует "шахматную" структуру, а основное течение представляет собой струю, перемежающуюся между твердыми продольными стенками диффузора. При увеличении числа Рейнольдса стационарные течения в диффузоре теряют устойчивость и переходят в колебательный режим течения, характеристики которого меняются по длине диффузора.

На рис. 9, $a$, б представлены изолинии горизонтальной компоненты скорости для всей области, а также в увеличенном масштабе для входного участка диффузора, отмеченного овалом. 
Здесь же на нижнем рисунке показаны изолинии угла вектора скорости.

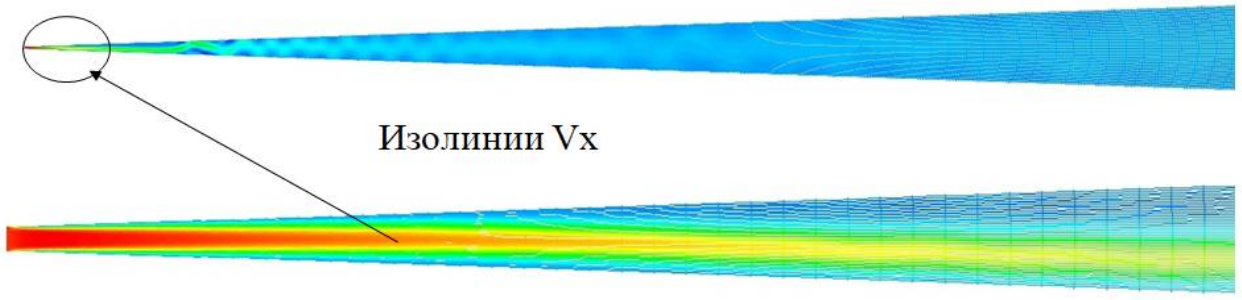

Изолинии Vx (входной участок диффузора)

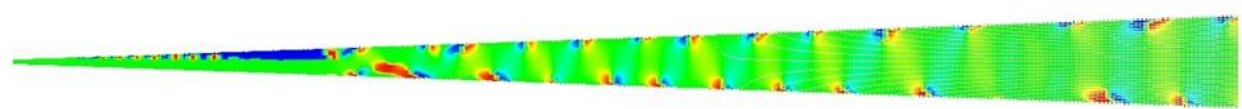

Изолинии угла вектора скорости $\operatorname{arctg}(\mathrm{Vx} / \mathrm{Vy})$

Рис. 9, $a$. Изолинии горизонтальной компоненты скорости (вверху) и угла вектора скорости (внизу) течения псевдопластичной жидкости в диффузоре $(Q=0.5[\kappa г / \mathrm{c}], \operatorname{Re}=500$, $n=0.5$ )

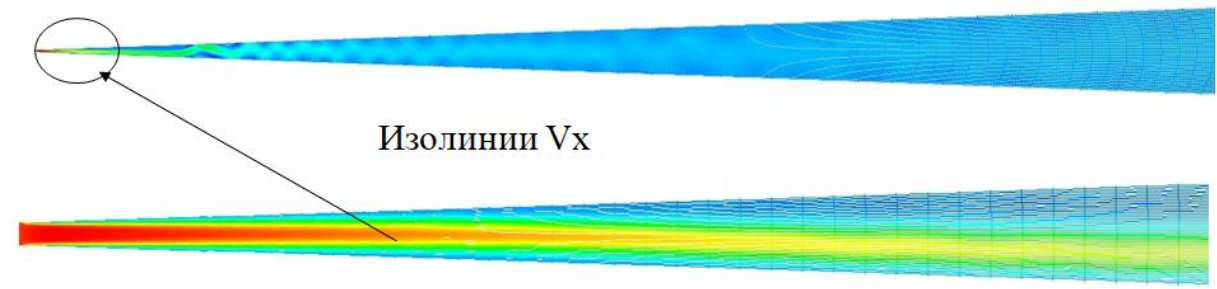

Изолинии Vx (входной участок диффузора)

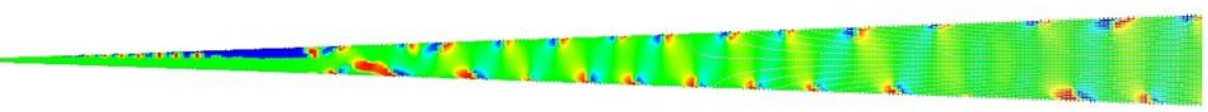

Изолинии угла вектора скорости $\operatorname{arctg}(\mathrm{Vx} / \mathrm{Vy})$

Рис. 9, б. Изолинии горизонтальной компоненты скорости (вверху) и угла вектора скорости (внизу) течения ньютоновской жидкости в диффузоре ( $Q=0.5[$ [г/с $], \operatorname{Re}=500, n=1)$

На рис. 10, $a$ и рис. 10, б представлены безразмерные профили модуля скорости в четыpex вертикальных сечениях $x=0.1,0.2,0.3,0.4$ м для течения псевдопластичной жидкости $(n=0.5)$ и ньютоновской жидкости $(n=1)$ в диффузоре при $\mathrm{Re}=500$.

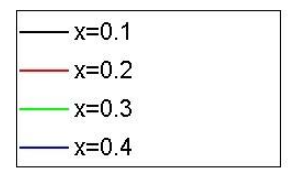

vmag_dimless

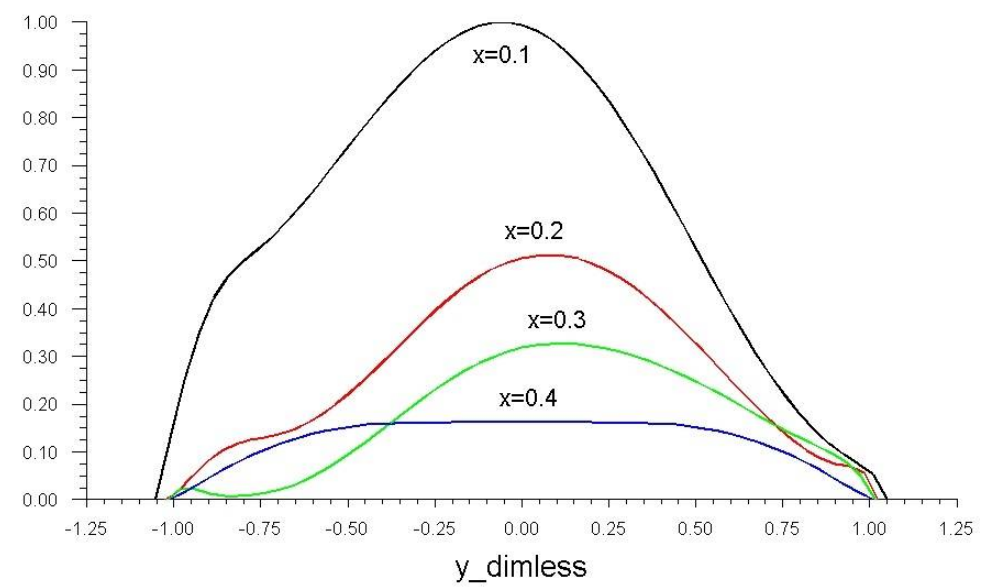

Рис. 10, $a$. Профили скорости течения псевдопластичной жидкости в диффузоре $(Q=0.5$ $[\kappa г / \mathrm{c}], \operatorname{Re}=500, n=0.5$, в сечениях $x=0.1,0.2,0.3,0.4$ м) 


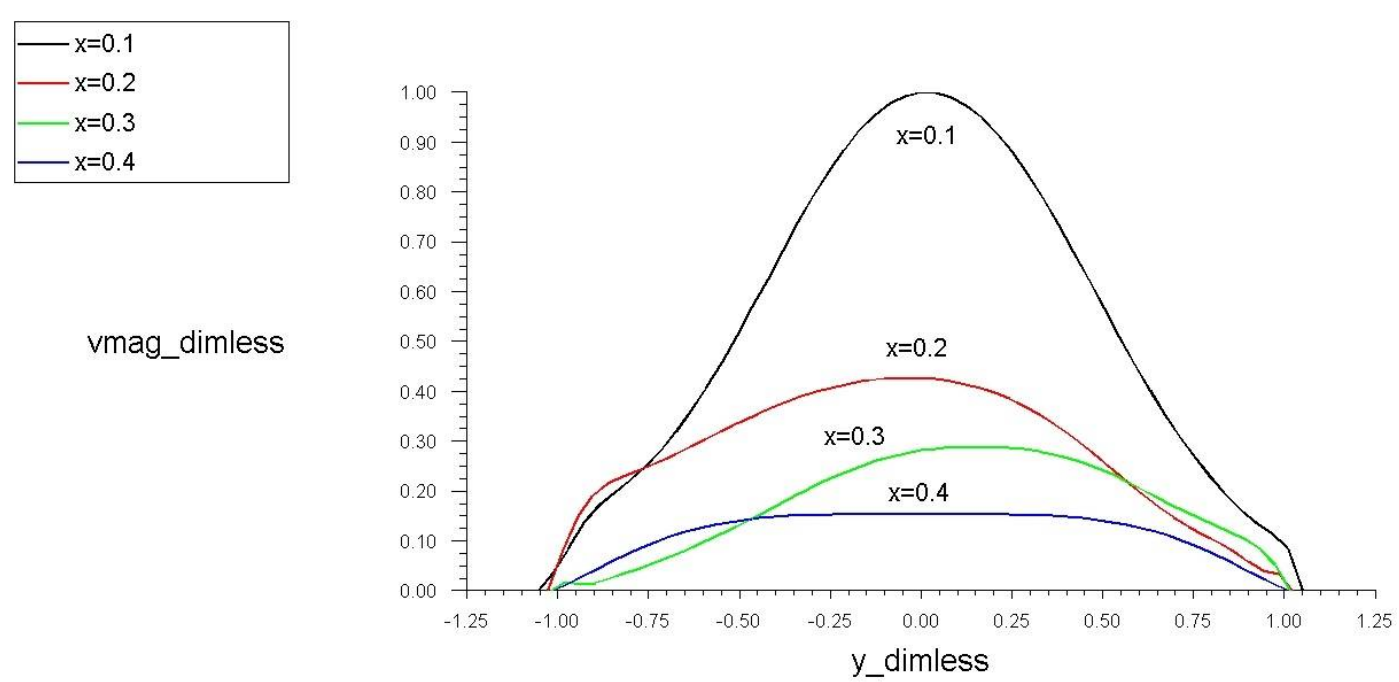

Рис. 10, б. Профили скорости течения ньютоновской жидкости в диффузоре $(Q=0.5$

$[\kappa г / \mathrm{c}], \operatorname{Re}=500, n=1$, в сечениях $x=0.1,0.2,0.3,0.4 \mathrm{м})$

Сравнение результатов, представленных на рис. 9 и рис. 10 говорит о том, что течения ньютоновской жидкости и псевдопластичной жидкости в диффузоре при одинаковых параметрах $(\operatorname{Re}=500)$ несимметричны и значительно различаются друг от друга на начальном участке диффузора и становятся близкими друг к другу и практически симметричными в конце диффузора. Результаты моделирования показали, что течение жидкости в конфузоре остается стационарным и симметричным при более больших числах Рейнольдса, чем в диффузоре.

\section{4. Течения с несимметричными начальными профилями скорости}

Рассмотрим влияние несимметричности граничных условий для скорости на входе в канал. В данном параграфе представлены результаты течения ньютоновской жидкости в конфузоре и диффузоре при несимметричных профилях скорости, заданных на входе в диффузор и конфузор. Рассматривается изменение несимметричного потока и установление симметричности течения ньютоновской жидкости в конфузоре и диффузоре для чисел Рейнольдса $\mathrm{Re}=100, \operatorname{Re}=500, \mathrm{Re}=1000 . \mathrm{B}$ начальный момент скорости во всей расчетной области были нулевыми.

Уравнение несимметричного профиля нормальной скорости на входе в диффузор задавалось выражением

$$
V_{\text {in }}=\frac{200 \cdot \operatorname{Re}\left(y+2 \times 10^{-4}\right) *\left(4 \times 10^{-8}-y^{2}\right)}{3}
$$

Уравнение несимметричного профиля нормальной скорости на входе в конфузор задавалось выражением

$$
V_{\text {in }}=\frac{200 \cdot \operatorname{Re}\left(y+1.74 \times 10^{-2}\right) *\left(3.03 \times 10^{-4}-y^{2}\right)}{3}
$$

Число Рейнольдса вычислялось по максимальной скорости на входе в диффузор (конфузор).

\subsection{1. Течение в конфузоре с несимметричным начальным профилем скорости}

На рис. 11-14 представлены безразмерные профили модуля скорости течения несжимаемой жидкости в конфузоре в разных поперечных сечениях (на входе в конфузор и при $x=0.1,0.2,0.3,0.4$ м) для чисел Рейнольдса $\mathrm{Re}=100, \mathrm{Re}=500, \mathrm{Re}=1000$, при заданных несимметричных граничных условиях для скорости $V_{\text {in }}$ на входе в конфузор. Профили скорости 
показывают восстановление симметричного течения в конфузоре при удалении от его входной границы.

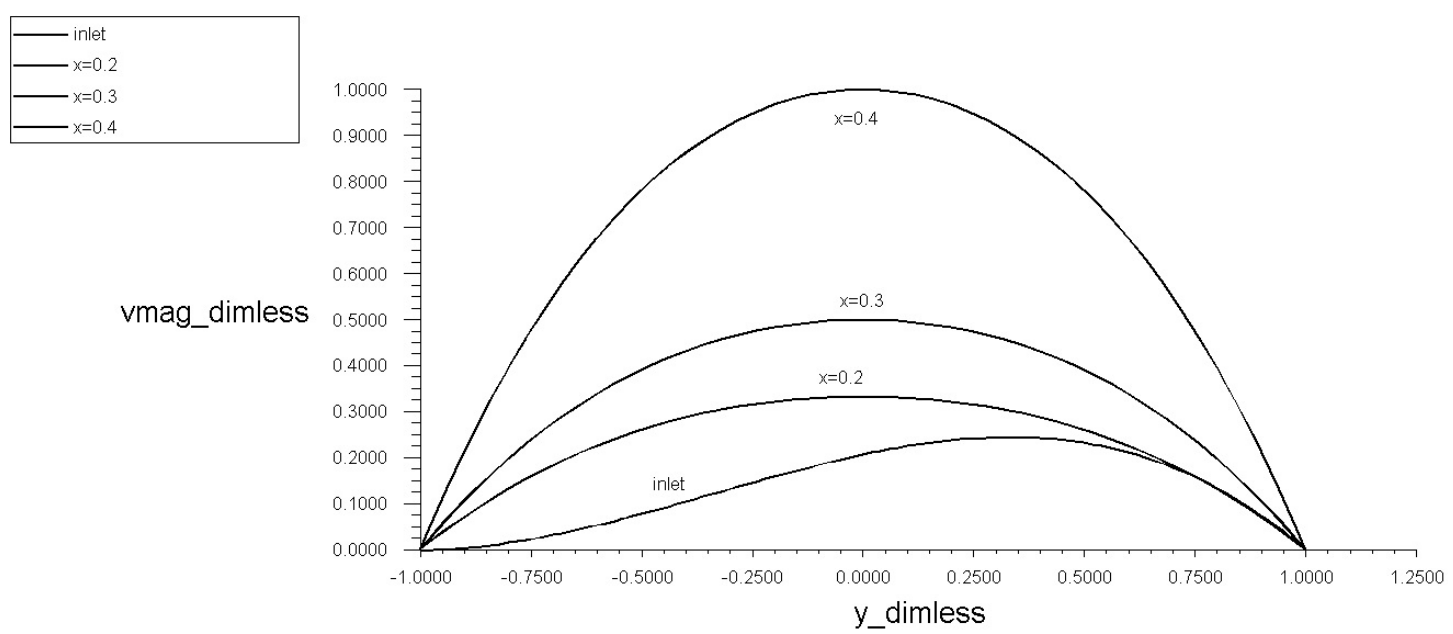

Рис. 11. Профили скорости течения ньютоновской жидкости в конфузоре ( $Q=0.1[$ кг/с], $\operatorname{Re}=100, n=1$, на входе и в сечениях $x=0.1,0.2,0.3,0.4$ м)
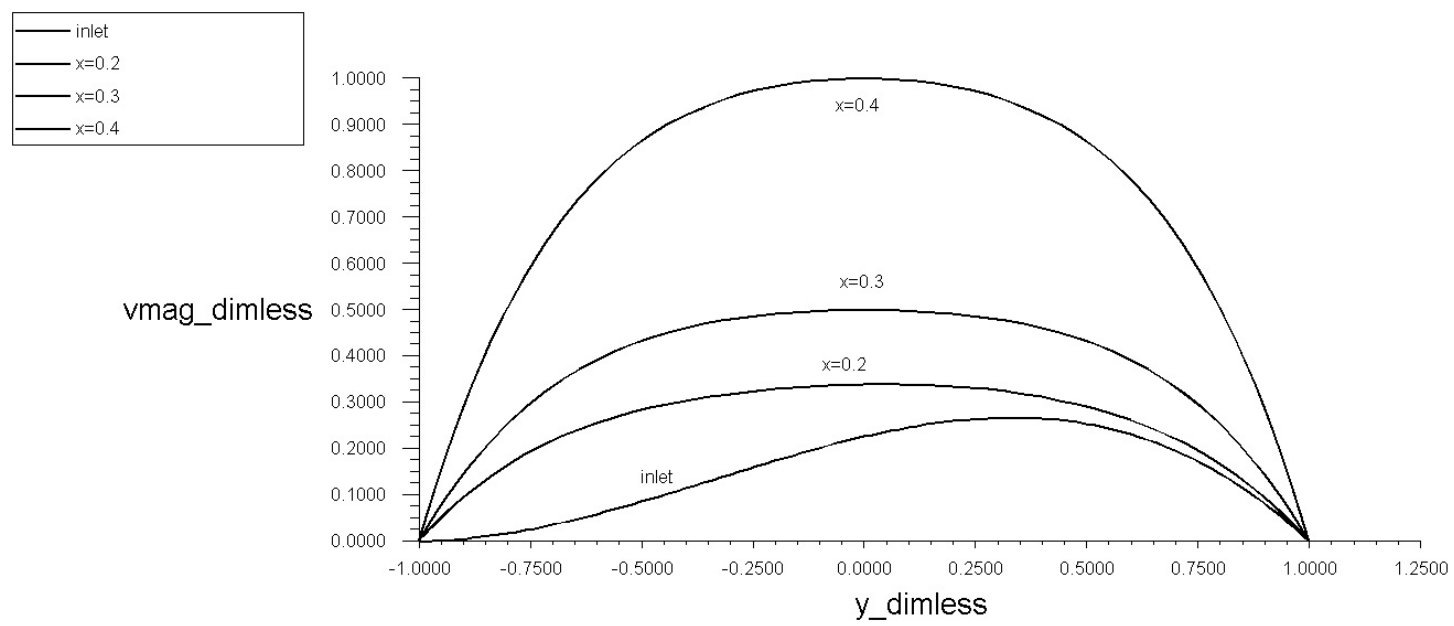

Рис. 12. Профили скорости течения ньютоновской жидкости в конфузоре ( $Q=0.5$ [кг/с], $\operatorname{Re}=500, n=1$, на входе и в сечениях $x=0.1,0.2,0.3,0.4$ м)

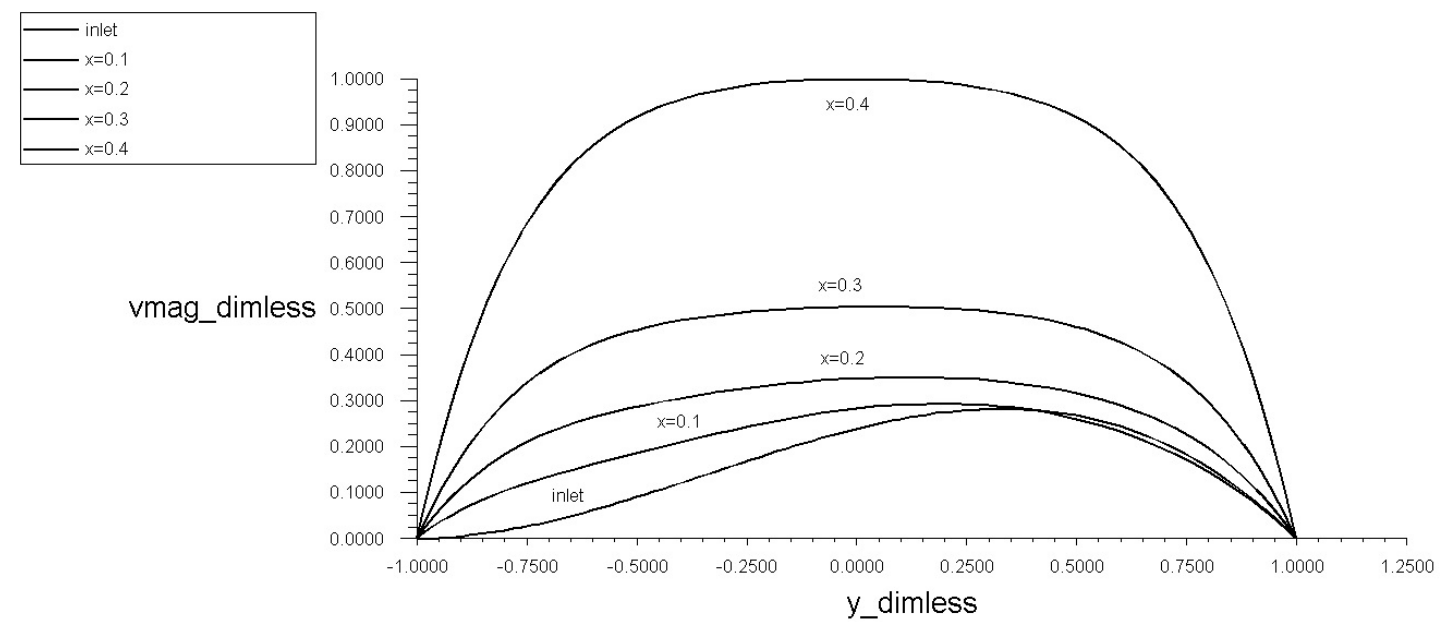

Рис. 13. Профили скорости течения ньютоновской жидкости в конфузоре ( $Q=1[$ кг/с], $\operatorname{Re}=1000, n=1$, на входе и в сечениях $x=0.1,0.2,0.3,0.4$ м) 
При числе Рейнольдса $\mathrm{Re}=3000$ поток в конфузоре остается несимметричным во всей расчетной области рис.14. Следует отметить, что при симметричных граничных условиях на входе в конфузор, течение в конфузоре оставалось симметричным.

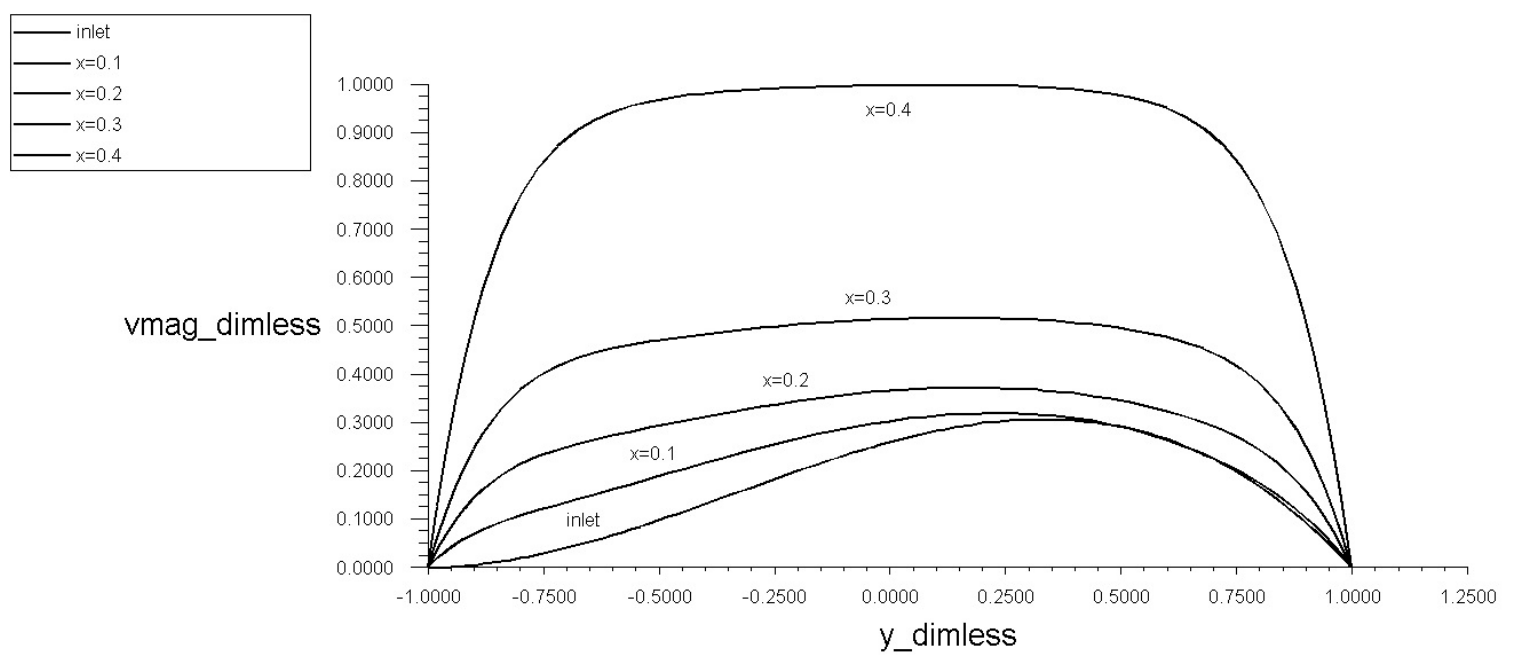

Рис. 14. Профили скорости течения ньютоновской жидкости в конфузоре ( $Q=3[$ [кг/с], $\operatorname{Re}=3000, n=1$, на входе и в сечениях $x=0.1,0.2,0.3,0.4 \mathrm{м}$ )

\subsection{2. Течение в диффузоре с несимметричным начальным профилем скорости}

Несимметричный профиль безразмерной скорости, задаваемый на входе в диффузор представлен на рис. 15. На рис. 16-18 представлены безразмерные профили модуля скорости течения несжимаемой жидкости в диффузоре в разных поперечных сечениях $(x=0.1,0.2,0.3$, 0.4 м) для чисел Рейнольдса $\mathrm{Re}=100, \mathrm{Re}=500, \mathrm{Re}=1000$, при заданных несимметричных граничных условиях для скорости $V_{\text {in }}$ на входе в диффузор.

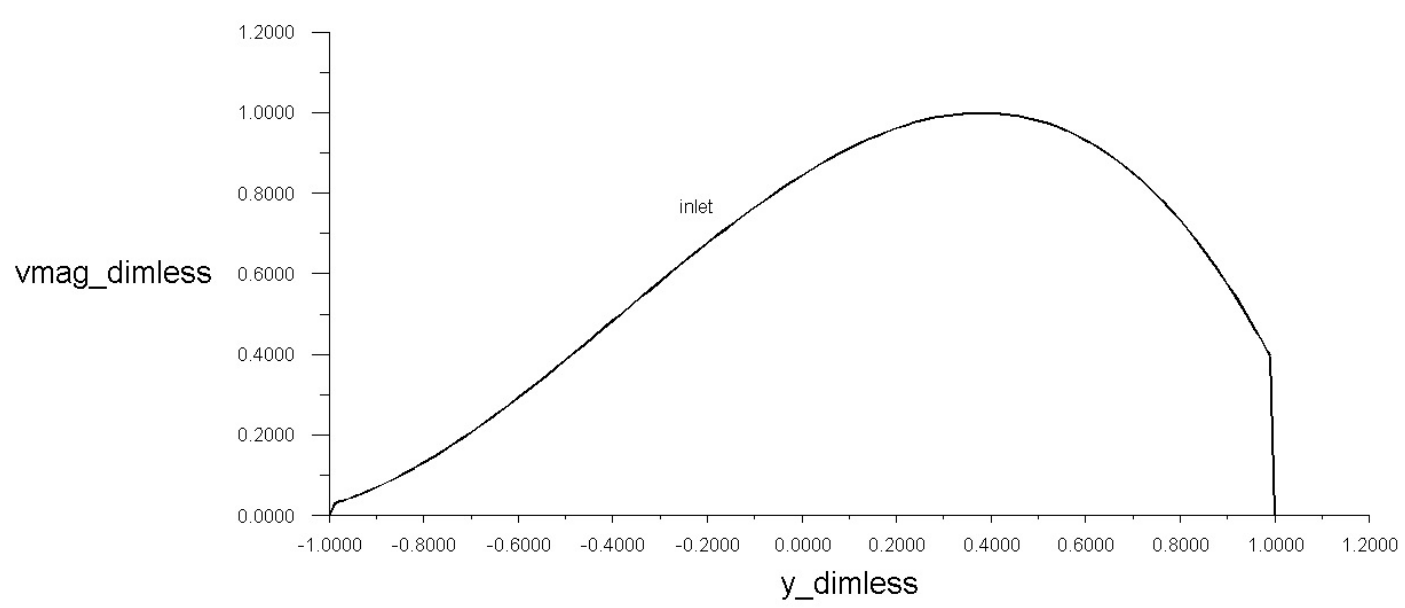

Рис. 15. Несимметричный профиль безразмерной скорости, задаваемый на входной границе в диффузор

Результаты численного моделирования с начальными несимметричными профилями скорости показали, что в диффузоре изначально несимметричный поток жидкости симметризуется, переходя от сечения к сечению по длине диффузора (в задаче ДГ скорость на бесконечности асимптотически стремится к нулю). В отличие от диффузора, в конфузоре симметризация потока идет значительно медленнее. 


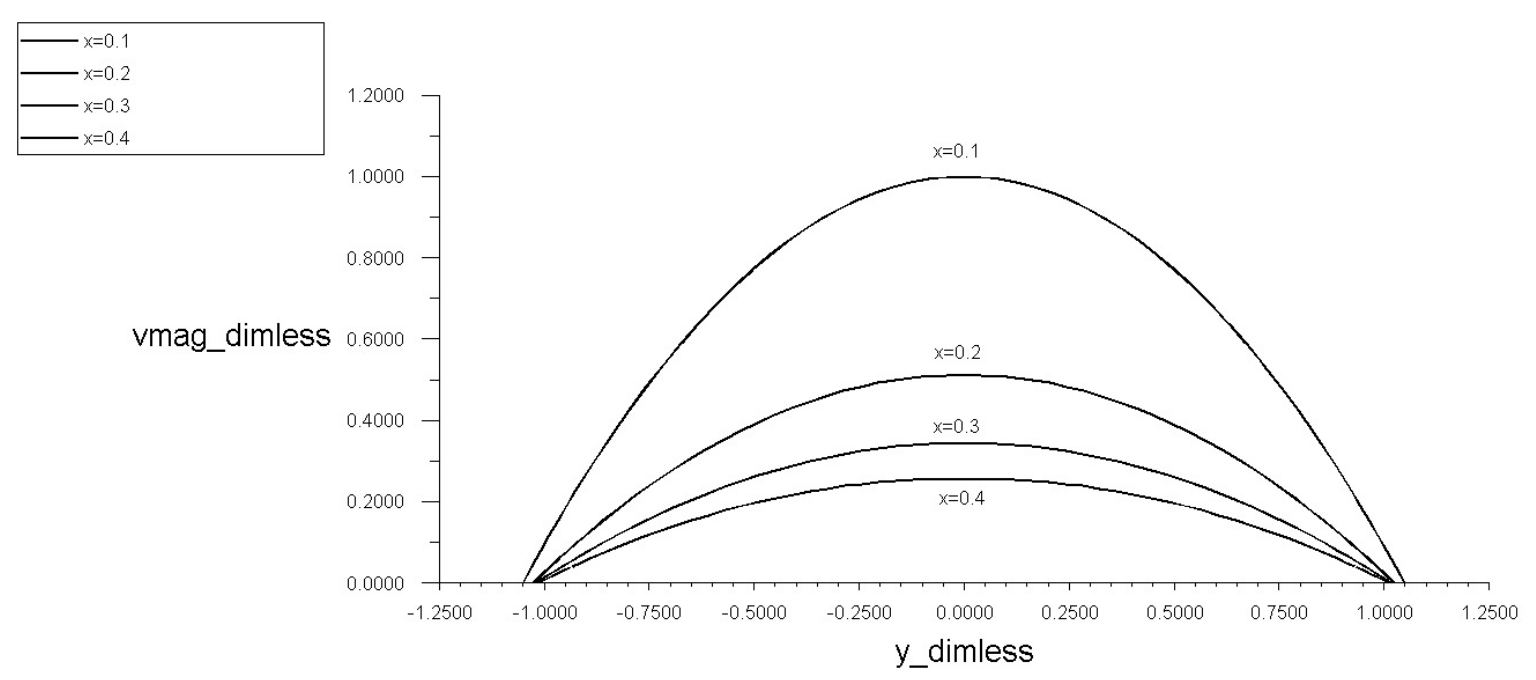

Рис. 16. Профили скорости течения ньютоновской жидкости в диффузоре ( $Q=0.1$ [кг/с], $\operatorname{Re}=100, n=1$, на входе и в сечениях $x=0.1,0.2,0.3,0.4$ м)

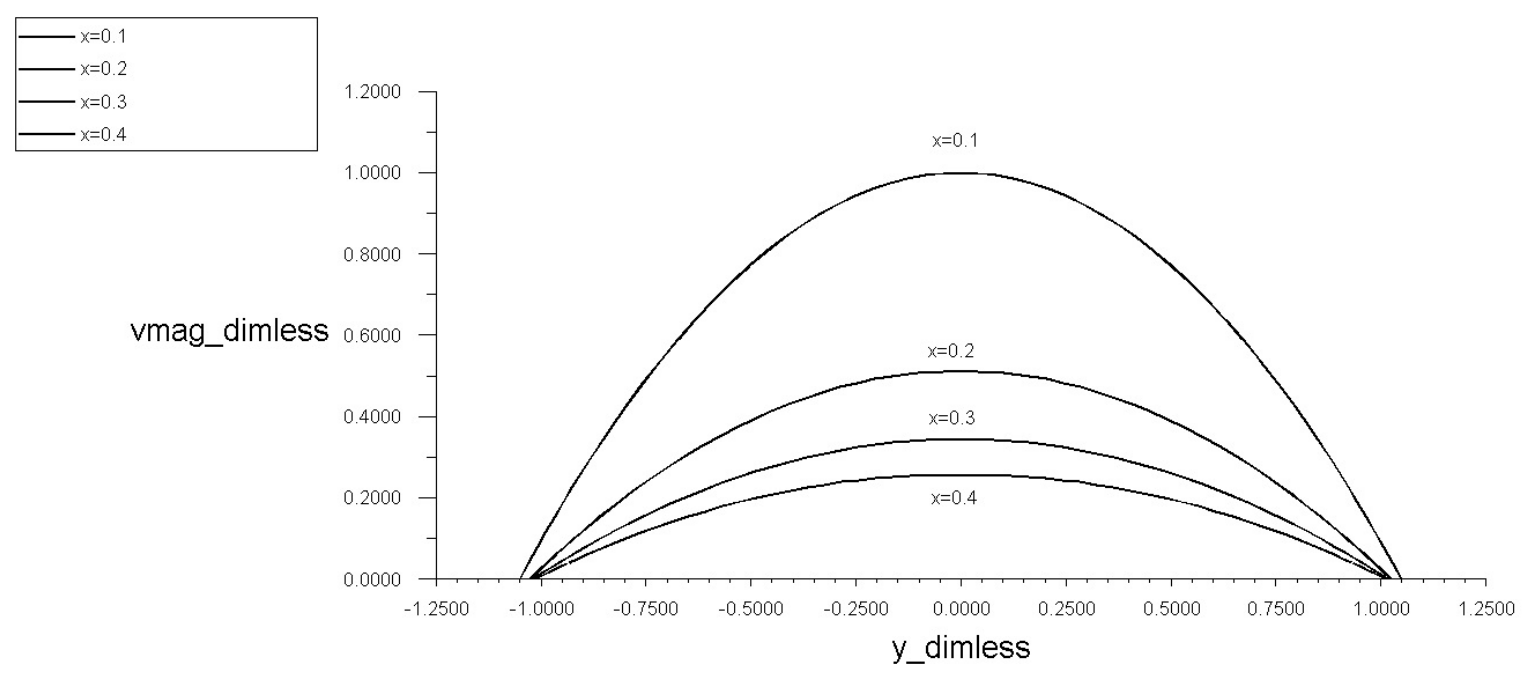

Рис. 17. Профили скорости течения ньютоновской жидкости в диффузоре ( $Q=1[$ [кг/с], $\operatorname{Re}=1000, n=1$, на входе и в сечениях $x=0.1,0.2,0.3,0.4 \mathrm{M}$ )
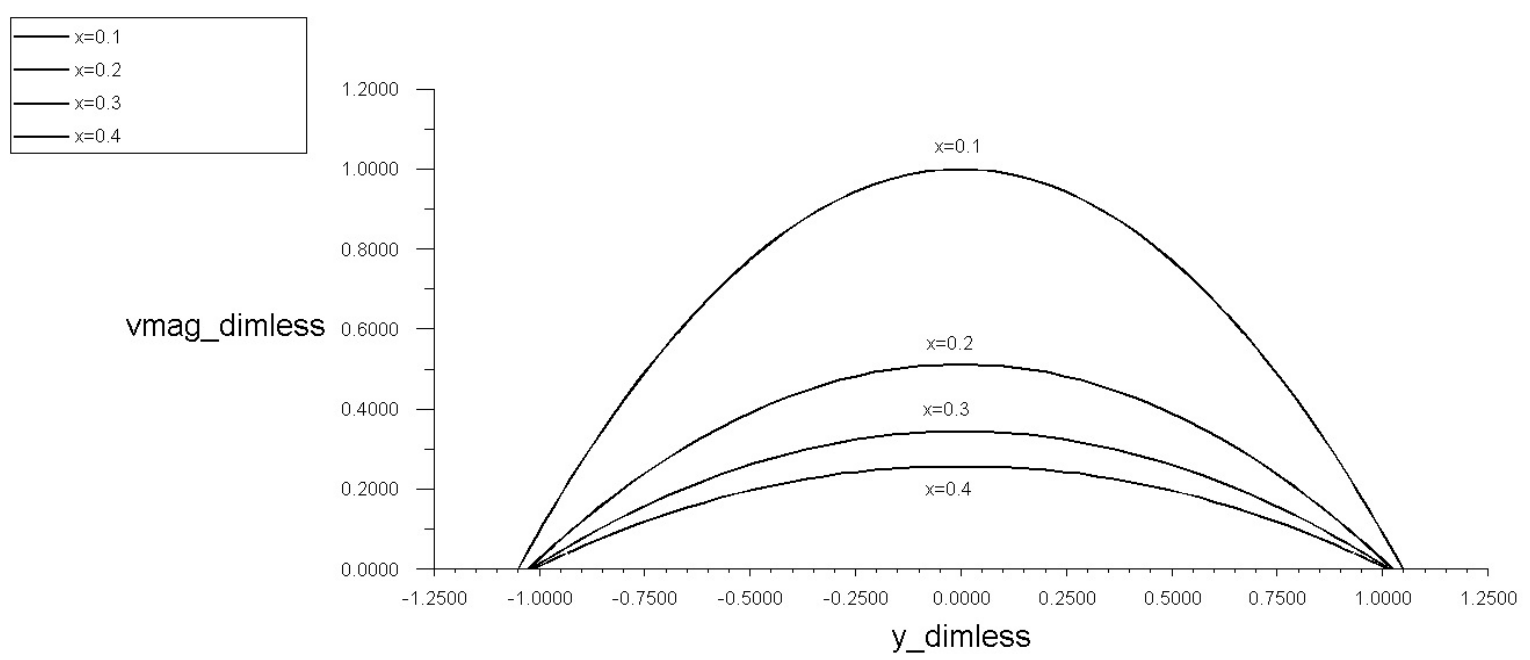

Рис. 18. Профили скорости течения ньютоновской жидкости в диффузоре ( $Q=3$ [кг/с], $\operatorname{Re}=3000, n=1$, на входе и в сечениях $x=0.1,0.2,0.3,0.4$ м) 


\section{4. Заключение}

Для течений псевдопластичных $(n=0.5)$ и ньютоновских $(n=1)$ жидкостей в плоском диффузоре численно показаны диапазоны чисел Рейнольдса существования стационарных симметричных, стационарных несимметричных и нестационарных несимметричных режимов течения. Численные результаты показали, что в диффузоре при увеличении числа Рейнольдса выше критического значения возникает неустойчивость пограничного слоя и на некотором расстоянии от входа в диффузор вблизи продольных твердых стенок диффузора образуются слабые возвратные потоки. Это наблюдается, как при течении псевдопластичной жидкости $(n=0.5)$, так и при течении ньютоновской жидкости $(n=1)$.

Численные расчеты показали, что в диапазоне чисел Рейнольдса $300>\mathrm{Re}>270$ течение, как псевдопластичной жидкости $(n=0.5)$, так и ньютоновской жидкости $(n=1)$ в диффузоре перестает быть симметричным, оставаясь стационарным. Для дилатантной жидкости $(n=2)$ в рассматриваемом диапазоне чисел Рейнольдса наблюдались только стационарные симметричные течения.

В рассматриваемом диапазоне чисел Рейнольдса $(\operatorname{Re}<500)$ в плоском конфузоре течение является стационарным и симметричным, стационарный режим с несимметричной структурой течения не наблюдается.

В диффузоре изначально несимметричный ламинарный поток жидкости при удалении от входа в канал симметризуется гораздо быстрее, чем в конфузоре.

\section{Литература}

1. Джеффри Дж. Б. Двумерное установившееся движение вязкой жидкости. Перевод с английского Д.В. Георгиевского (соредакторы перевода Л.Д. Акуленко, С.В. Нестеров). // Нелинейная динамика, 2009, Т.5, №1, 2009г., С.101-109.

2. Гамель Г. “Спиралевидные движения вязкой жидкости”, Перевод с немецкого С.В. Нестерова (соредакторы перевода Л.Д. Акуленко, Д.В. Георгиевский). // Нелинейная динамика, Т.5, № 1, 2009г., С.111-133.

3. Кочин Н.Е., Кибель И.А., Розе Н.В. Теоретическая гидродинамика, ч.II, Москва: Физматгиз, $1963.728 \mathrm{c}$.

4. Бэтчелор Дж. Введение в динамику жидкости. М.: Мир, 1973г. 758 с.

5. Пухначев В.В. Симметрии в уравнениях Навье-Стокса // Успехи механики. 2006г.

6. Rosenhead L. The steady two-dimensional radial flow of viscous fluid between two inclined plane walls. Proc Roy Soc London Ser A 1940, vol.175, N 963, pp. 436-467.

7. Акуленко Л.Д., Георгиевский Д.В., Кумакшев С.А. Регулярно продолжаемые по числу Рейнольдса решения задачи Джеффри -Гамеля. //Изв. РАН. МЖГ. 2004г. № 1. С. 15-32.

8. Akulenko, L.D. \& Kumakshev, S.A. 2008 Bifurcation of multimode flows of a viscous fluid in a plane diverging channel. J. Appl. Math. Mech. 72(3), pp. 296-302.

9. M. Goldshtik, F. Hussain and V. Shtern. Symmetry breaking in vortex-source and Jeffery-Hamel flows. J. Fluid mech. (1991), vol. 232, pp. 521-566.

10. F. Durst, A. Melling, J.H. Whitelaw. Low Reynolds number flow over a plane symmetric sudden expansion, Journal of Fluid Mechanics, vol. 64, Issue 01, June 1974, pp 111-128.

11. D. Drikakis, Bifurcation phenomena in incompressible sudden expansion flows. Phys. Fluids, 9, 1997, pp.76-86.

12. M. Thiruvengadam, B.F. Armaly, J.A. Drallmeier. Three dimensional mixed convection in plane symmetric-sudden expansion. Three dimensional mixed convection in plane symmetric-sudden expansion: Symmetric flow regime. Int. Journal of Heat and Mass Transfer, 52, 2009, pp. 899-907.

13. T. Mullin and K. A. Cliffe (1986), Symmmetry breaking and the onset of time dependence in fluid mechanical systems, in Nonlinear Phenomena and Chaos (S. Sarkar, ed.), Hilger, London, pp. 96-112 
14. K.A. Cliffe, A. Spence, S.J. Tavener. The numerical analysis of bifurcation problems with application to fluid mechanics. Acta Numerica (2008), pp. 39-131.

15. Tutty O.R. Nonlinear development of flow in channels with non-parallel walls. J. Fluid Mech. (1996), vol. 326, pp. $265-284$.

16. F. Battaglia, S.J. Tavenery, A.K. Kulkarniz, C.L. Merklex. Bifurcation of low Reynolds number flows in symmetric channels. 1997, AIAA J. 35, 99-105

17. P.E. Haines, R.E. Hewitt, A.L. Hazel. The Jeffery-Hamel similarity solution and its relation to flow in a diverging channel. J. Fluid Mech. (2011), vol. 687, pp. 404-430.

18. Majid Nabavi. Three-dimensional asymmetric flow through a planar diffuser: Effects of divergence angle, Reynolds number and aspect ratio. Int. Communications in Heat and Mass Transfer 37, 2010, pp. 17-20.

19. Turkyilmazoglu M. Extending the traditional Jeffery-Hamel flow to stretchable convergent/divergent channels. Comput Fluids 2014; 100:196-203.

20. Puneet Rana, Nisha Shukla, Yogesh Gupta, Ioan Pop. Homotopy analysis method for predicting multiple solutions in the channel flow with stability analysis. Commun Nonlinear Sci Numer Simulat 66 (2019) 183-193.

21. Федоренко А.Т. Численное исследование нестационарных дозвуковых течений вязкого газа во внезапно расширяющемся плоском канале. // Изв. РАН. МЖГ. 1988. № 4. С. 32-41.

22. Федюшкин А.И. Переход течений вязкой несжимаемой жидкости в плоском диффузоре от симметричного к несимметричному и к нестационарному режимам. Рецензируемый электронный журнал «Физико-химическая кинетика в газовой динамике». 2016г., Т.17, вып. 3, 21с.

23. H.K. Versteeg, W. Malalasekera - An Introduction to Computational Fluid Dynamics. The Finite Volume Method. -Second edition. -England., 2007, pp. 115-121.

24. Рейнер М. Реология. М.: Мир, 1965. 224 с.

25. Шульман 3.П. Конвективный тепломассоперенос реологически сложных жидкостей. М., “Энергия”. 1975г. 352 с. 